\title{
X-ray spectral diagnostics of activity in massive stars
}

\author{
David H. Cohen ${ }^{1}$, Emma E. Wollman ${ }^{2}$ and Maurice A. Leutenegger ${ }^{3}$ \\ ${ }^{1}$ Department of Physics and Astronomy, Swarthmore College, \\ 500 College Ave., Swarthmore, Pennsylvania, 19081, USA \\ email: cohen@astro.swarthmore.edu \\ ${ }^{2}$ Department of Physics, California Institute of Technology, \\ Pasadena, California, 91125, USA \\ email: ewollman@caltech.edu \\ ${ }^{3}$ NASA/Goddard Spaceflight Center, Code 662, Greenbelt, Maryland, 20771, USA \\ email: maurice.a.leutenegger@nasa.gov
}

\begin{abstract}
X-rays give direct evidence of instabilities, time-variable structure, and shock heating in the winds of $\mathrm{O}$ stars. The observed broad X-ray emission lines provide information about the kinematics of shock-heated wind plasma, enabling us to test wind-shock models. And their shapes provide information about wind absorption, and thus about the wind mass-loss rates. Mass-loss rates determined from X-ray line profiles are not sensitive to density-squared clumping effects, and indicate mass-loss rate reductions of factors of 3 to 6 over traditional diagnostics that suffer from density-squared effects. Broad-band X-ray spectral energy distributions also provide mass-loss rate information via soft X-ray absorption signatures. In some cases, the degree of wind absorption is so high, that the hardening of the X-ray SED can be quite significant. We discuss these results as applied to the early O stars $\zeta$ Pup (O4 If), 9 Sgr (O4 V((f))), and HD 93129A (O2 If*).
\end{abstract}

Keywords. line: formation, shock waves, stars: winds, outflows, X-rays: stars

\section{Introduction}

Soft X-ray emission is ubiquitous in O stars, and it is generally accepted that it arises in numerous shock-heated regions embedded in these stars' powerful and dense radiationdriven winds. The broad emission lines seen in high-resolution X-ray spectra of $\mathrm{O}$ stars confirm this scenario qualitatively. In this paper, we present quantitative analysis of resolved X-ray emission lines observed in three very early $\mathrm{O}$ stars, from which we are able to place constraints on the kinematics and spatial distribution of the shock-heated plasma and thereby test predictions of numerical simulations of wind shocks. We also show how the degree of attenuation by the bulk wind in which the shocked plasma is embedded can be measured both from resolved emission lines and from lower resolution broadband X-ray spectra in order to estimate the mass-loss rates of $\mathrm{O}$ star winds.

We restrict our discussion to "normal" massive stars, where binarity and the associated colliding wind shock (CWS) X-ray emission and magnetically channeled wind shock (MCWS) X-ray emission is absent or negligible. The dominant paradigm for X-ray production in normal $\mathrm{O}$ and early-B stars is the embedded wind shock (EWS) scenario, and the specific mechanism for EWSs is usually assumed to involve the line-driving instability (LDI), either in a self-excited mode (Owocki, Castor, \& Rybicki 1988) or in a mode where the instability is seeded by perturbations at the base of the wind (Feldmeier, Puls, \& Pauldrach 1997). 

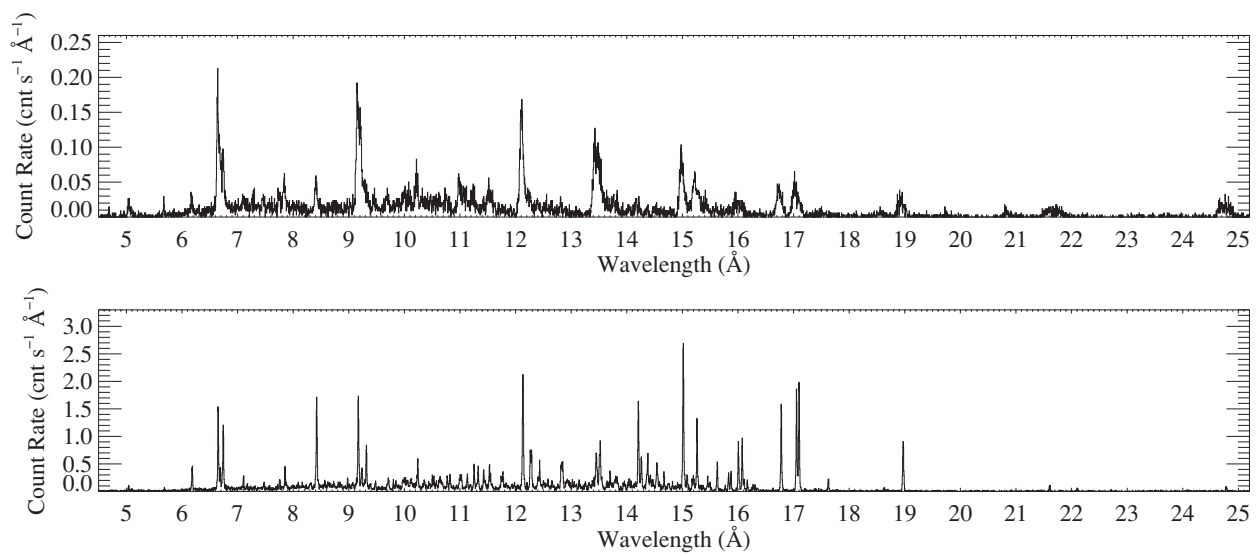

Figure 1. Chandra medium energy grating (MEG) spectra of the O4 If star, $\zeta$ Pup (top) and for comparison, of the G star, Capella (bottom). The spectrum of the $\mathrm{O}$ star is harder (strongest lines at shortest wavelengths) but by comparing H-like and He-like line strengths (e.g. of $\mathrm{Mg}$ at $8.42 \AA$ and $9.2 \AA$, respectively) it is evident that the higher temperature plasma is found on the $\mathrm{G}$ star. Finally, note that the emission lines are unresolved in the Capella spectrum and are significantly broadened in the $\zeta$ Pup spectrum.

The morphology of high-resolution X-ray spectra of normal massive stars reveals some important qualitative properties of $\mathrm{O}$ star X-rays. In Fig. 1 we compare the $\mathrm{O}$ supergiant $\zeta$ Pup to the coronal G star, Capella, to highlight some of these properties. The O star's spectrum is harder, overall, than the G star's, however this is due not to higher plasma temperatures, but rather to the effects of wind absorption, consistent with the X-rays arising in the dense stellar wind of the $\mathrm{O}$ star. This broadband view of the X-ray spectra also shows quite obviously that the emission lines in the $\mathrm{O}$ star are much broader than the (unresolved) lines in the G star, as the EWS scenario predicts. In the next section, we show how quantitative information can be derived from the Doppler broadened X-ray emission lines.

\section{The X-ray line profile model applied to $\zeta$ Pup}

To extract information from individual resolved line profiles, we fit a simple windshock model informed by the LDI simulations, in which numerous shock-heated regions are distributed throughout the wind above some shock onset radius, $R_{0}$, with local emission measure assumed to scale with the local ambient wind density squared (Owocki \& Cohen 2001). The kinematic profile of the X-ray plasma is assumed to trace the same beta-velocity law that describes the bulk wind. This assumption is based on the results of numerical simulations of EWSs that show accelerated pre-shock wind streams being decelerated back down the local ambient wind velocity (Runacres \& Owocki 2002). The attenuation due to continuum opacity in the bulk wind in which the shock-heated plasma is embedded is described by the characteristic optical depth parameter, $\tau_{*} \equiv \kappa \dot{M} / 4 \pi R_{*} v_{\infty}$, where $\kappa$ is the (wavelength dependent) opacity of the bulk wind, $\dot{M}$ is the wind mass-loss rate, $R_{*}$ is the stellar radius, and $v_{\infty}$ is the wind terminal velocity. The absorption of $\mathrm{X}$-rays imparts a characteristic blue-shifted and asymmetric shape to the emission line profiles due to the preferential attenuation of red-shifted line photons emitted in the far hemisphere of the wind, while leaving blue-shifted line photons emitted from the near hemisphere much less attenuated.

For each emission line in the Chandra spectrum of an $\mathrm{O}$ star, we can fit this empirical profile model and derive best-fit values of $R_{\mathrm{o}}$ and $\tau_{*}$ by minimizing the $\mathrm{C}$ statistic, and 

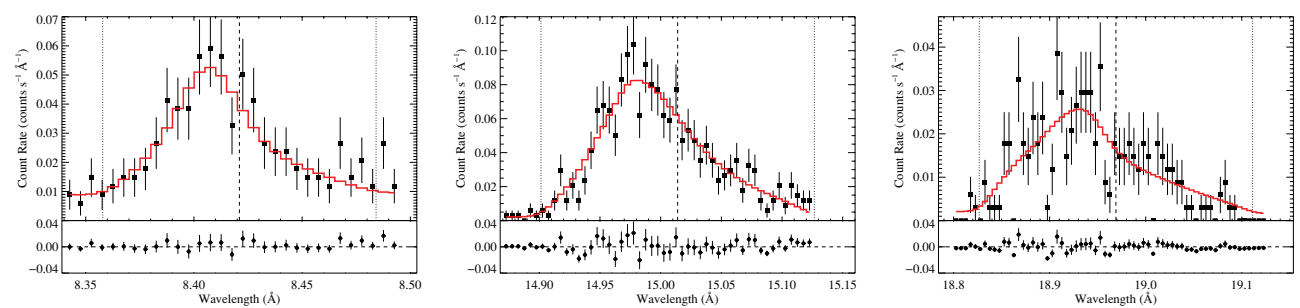

Figure 2. Fits to three lines in the Chandra spectrum of $\zeta$ Pup. From left to right: the Ly $\alpha$ line of $\mathrm{Mg}$ XII at $8.42 \AA$, the Fe XVII line at $15.01 \AA$, and the Ly $\alpha$ line of O VIII at 18.97 $\AA$. The vertical dashed lines in each panel represent the laboratory rest wavelengths of each transition, while the flanking dotted lines represent the Doppler shifts associated with the wind terminal velocity. The characteristic broad, blue shifted, and asymmetric profile shapes are evident, as is an increase in the shift and asymmetry with wavelength, as is expected from the form of the continuum opacity of the bulk wind, which generally increases with wavelength. The characteristic optical depths of these three lines are roughly $\tau_{*}=1,2$, and 3 , respectively.

place confidence limits on them via the $\Delta \chi^{2}$ formalism applied to the $\mathrm{C}$ statistic. For $\zeta$ Pup (summarizing the results published in Cohen et al. 2010), we find - for 16 lines and line complexes in the Chandra grating spectrum (three representative lines and their best-fit profile models are shown in Fig. 2) - a universal value for the shock-onset radius of $R_{\mathrm{o}} \approx 1.5 \mathrm{R}_{*}$, which is consistent with numerical simulations of the LDI (Feldmeier, Puls, \& Pauldrach 1997, Runacres \& Owocki 2002). We also find a range of characteristic optical depths, $\tau_{*}$, for the 16 emission lines, consistent with the expected wavelength trend in the atomic opacity. By calculating a detailed opacity model, and assuming standard values for the stellar radius and wind terminal velocity, we fit the ensemble of characteristic optical depths to find a best-fit mass-loss rate, via $\dot{M}=4 \pi R_{*} v_{\infty} \tau_{*}(\lambda) / \kappa(\lambda)$. The values of the onset radius, $R_{0}$, and of the characteristic optical depths, $\tau_{*}$, are shown in Fig. 3. The panel with the $\tau_{*}$ values also shows the best-fit model of the wavelengthdependent optical depths, from which we derive a mass-loss rate of $3.5 \pm 0.3 \times 10^{-6}$ $\mathrm{M}_{\odot} \mathrm{yr}^{-1}$.

We emphasize that the mass-loss rate determination from the X-ray profiles represents a factor of roughly three reduction from the traditional $\mathrm{H} \alpha$-derived mass-loss rate that ignores clumping (Markova et al. 2004). And that this modest reduction in the mass-loss rate is consistent with newer determinations using $\mathrm{H} \alpha$ and radio and IR free-free excesses that do account for clumping (Puls et al. 2006). Also, we note that for a high signalto-noise Chandra spectrum with many emission lines, like that of $\zeta$ Pup, the statistical error on the derived mass-loss rate is small (about 10\%), but that the actual uncertainty is dominated by uncertainty in the wind opacity model, which in turn is dominated by uncertainty in the elemental abundances. Individual elemental abundances do not have a large effect, but the overall metallicity does. The model we use in this paper (and which was used in Cohen et al. (2010)) uses subsolar metallicity and C, N, and O abundances altered by CNO processing. If future abundance determinations are made which supersede the current ones, the mass-loss rate should be rescaled in inverse proportion to the metallicity adjustment (more metals cause higher opacity which would then require lower wind column densities and so lower mass-loss rates).

\section{Other O stars}

We can apply the same type of line profile analysis to other O stars observed with the Chandra grating spectrometer. Here we present preliminary analysis of the early $\mathrm{O}$ 

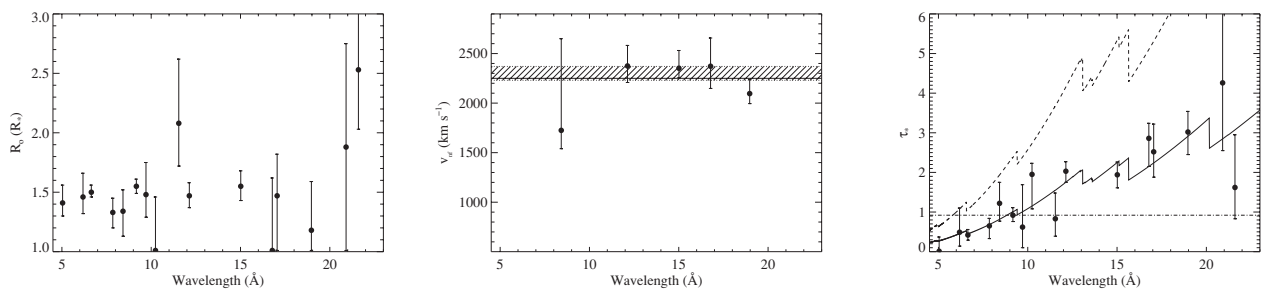

Figure 3. Results from fitting the wind-profile model to the emission lines in the Chandra spectrum of $\zeta$ Pup. From left to right: the shock onset radii, $R_{0}$, which are consistent with a universal value of $1.5 \mathrm{R}_{*}$; the wind terminal velocities for the five strongest, unblended lines in the spectrum, which are consistent with the value for the bulk wind, of $v_{\infty}=2250 \mathrm{~km} \mathrm{~s}^{-1}$, derived from UV spectra (this value is represented by the horizontal line, while the cross hatched region is the $68 \%$ confidence limit on the mean value of the five fitted terminal velocities shown as points with error bars); and the $\tau_{*}$ values from each of the 16 fitted line profiles. This last panel shows that a constant value of the characteristic optical depth provides a poor fit, as does a model that incorporates the continuum opacity of the bulk wind but assumes a high value for the mass-loss rate $\left(8.3 \times 10^{-6} \mathrm{M}_{\odot} \mathrm{yr}^{-1}\right)$, while a model with the mass-loss rate as a free parameter provides a good fit, with a mass-loss rate of $3.5 \times 10^{-6} \mathrm{M}_{\odot} \mathrm{yr}^{-1}$.

main sequence star, $9 \mathrm{Sgr}$, at the center of the Lagoon Nebula, and the very early O supergiant, HD 93129A, in Tr 14 in Carina. Both stars have binary companions, but in neither case are the emission lines in the grating spectrum significantly contaminated by the harder emission associated with CWB X-rays.

There are nine lines and line complexes in the 9 Sgr Chandra grating spectrum with high enough signal-to-noise for line profile modeling to provide meaningful constraints. We assume a wind velocity parameter $\beta=0.7$ and find a mean shock onset radius of $R_{\mathrm{o}}=1.4 \mathrm{R}_{*}$, consistent with the EWS scenario. The ensemble of $\tau_{*}$ values can be fit, given a model of the bulk wind opacity (which we calculate assuming solar abundances), to derive a mass-loss rate. We find a mass-loss rate of $\dot{M}=3.4 \times 10^{-7} \mathrm{M}_{\odot} \mathrm{yr}^{-1}$, which represents a factor of six reduction over the traditional mass-loss rate derived from $\mathrm{H} \alpha$ and radio free-free emission, assuming a smooth wind (Lamers \& Leitherer 1993, Puls et al. 1996). In Fig. 4 we show the $R_{\mathrm{o}}$ and $\tau_{*}$ results.

The O2 If* star, HD 93129A, is the earliest O star in the Galaxy and, according to Taresch et al. (1997), has the highest mass-loss rate of any O star, with $\dot{M}=1.8 \times 10^{-5}$ $\mathrm{M}_{\odot} \mathrm{yr}^{-1}$. More recent modeling (though also ignoring clumping effects) gives $\dot{M}=2.6 \times$ $10^{-5} \mathrm{M}_{\odot} \mathrm{yr}^{-1}$ and a wind terminal velocity of $v_{\infty}=3200 \mathrm{~km} \mathrm{~s}^{-1}$ (Repolust, Puls, \& Herrero 2004). This extremely strong and dense stellar wind provides an interesting test of the EWS scenario for X-ray production in O stars. Indeed, the Chandra spectrum is quite hard, which if assumed to be due to high temperature would make an EWS interpretation implausible. However, the H-like Si line strength is very weak, compared to the He-like Si line strength, indicating a plasma emission temperature of no more than 8 million $\mathrm{K}$, which is consistent with LDI simulations of wind shocks. The hardness of the X-ray spectrum appears instead to be due to severe attenuation of the soft X-ray emission by both the interstellar medium and the star's own wind.

Because of the absent soft X-rays, there are only four lines and line complexes in the Chandra grating spectrum available for fitting. We show the $\tau_{*}$ results in Fig. 4 with the mass-loss rate fit superimposed. For this star, too, we find a modest mass-loss rate reduction of roughly a factor of four over the value derived from $\mathrm{H} \alpha$ fitting assuming no clumping. 

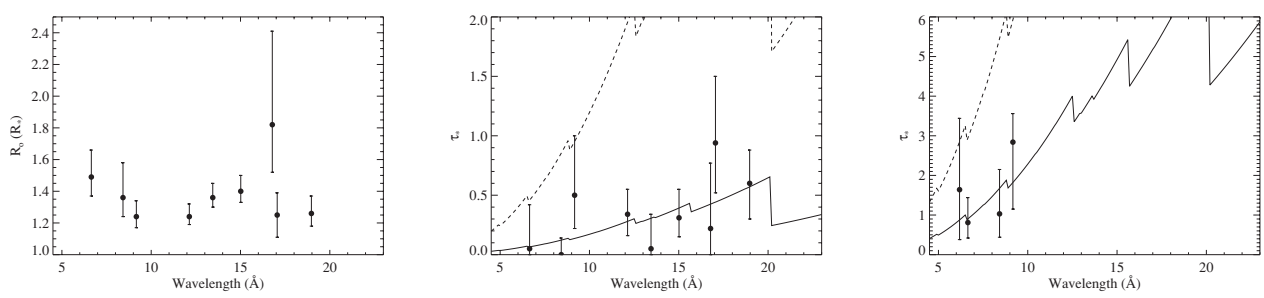

Figure 4. Results from fitting the wind-profile model to the emission lines in the Chandra spectrum of $9 \mathrm{Sgr}$ and HD 93129A. The shock onset radii for $9 \mathrm{Sgr}$ (left) are consistent with a value of $R_{0}=1.4 R_{*}$, while the characteristic optical depths (center) are well fit by a model that has a mass-loss rate of $\dot{M}=3.4 \times 10^{-7} \mathrm{M}_{\odot} \mathrm{yr}^{-1}$ (solid line), a factor of six below the unclumped $\mathrm{H} \alpha$ mass-loss rate (dotted line). For HD 93129A we show the $\tau_{*}$ values (right) along with the best-fit mass-loss rate model (solid line; $\dot{M}=6.8 \times 10^{-6} \mathrm{M}_{\odot} \mathrm{yr}^{-1}$ ) and for comparison, the traditional unclumped $\mathrm{H} \alpha$ mass-loss rate model. The opacity model used for HD 93129A assumes altered CNO abundances (Taresch et al. 1997).

\section{Broadband X-ray properties}

Given the strong wind absorption in the X-ray spectra of early O stars, we have modeled the broadband spectral energy distributions using simple one- and two-temperature thermal emission spectral models (e.g. APEC Smith et al. 2001) along with wind attenuation, using the newly published radiation transport model, windtabs (Leutenegger et al. 2010). This model accounts for the spatial distribution of the emitting plasma within the absorbing wind, and thus has a much more gradual decrease of transmission vs. fiducial optical depth than is seen in the exponential absorption model that describes an absorbing medium in between the background emitter and the observer, such as those employed in interstellar absorption models. The windtabs absorption model also uses a realistic photoionization opacity model that includes partially ionized metals and fully ionized $\mathrm{H}$ and He. We fit the Chandra zeroth-order spectrum (a CCD low-resolution spectrum) of HD 93129A with this APEC and windtabs model and find a low plasma temperature of $0.6 \mathrm{keV}$ - fully consistent with the LDI simulation results - and a significant wind column density, corresponding to a mass-loss rate of $\dot{M}=8 \times 10^{-6} \mathrm{M}_{\odot} \mathrm{yr}^{-1}$, which is consistent with the value we find from fitting the individual line profiles (shown in the third panel of Fig. 4). We show the HD 93129A zeroth-order spectrum and best-fit APEC and windtabs model in Fig. 5, along with the grating spectrum of the star, in which the low $\mathrm{H}$-like/He-like line ratios, indicative of low plasma temperatures, can be seen.

\section{Conclusions}

The X-ray emission from normal massive stars can be understood in the context of embedded wind shocks due to the line-driving instability. Specifically, the X-ray emitting plasma shares the same kinematic profile as the bulk wind. It is spatially distributed throughout the wind above an onset radius of roughly $R_{\mathrm{o}}=1.5 \mathrm{R}_{*}$ and - from broadband modeling - the plasma temperatures are less than 10 million $\mathrm{K}$, in accord with the predictions of LDI simulations of EWSs. These relatively low temperatures can be reconciled with the relatively hard observed spectra by taking wind attenuation of the soft X-rays into account. When we model the effect of wind attenuation on individual emission lines, we find that their modestly blue-shifted and asymmetric profiles can be reproduced using mass-loss rates that are lower by a factor of 3 to 6 compared to traditional mass-loss rates that ignore clumping (and are consistent with newer determinations that 

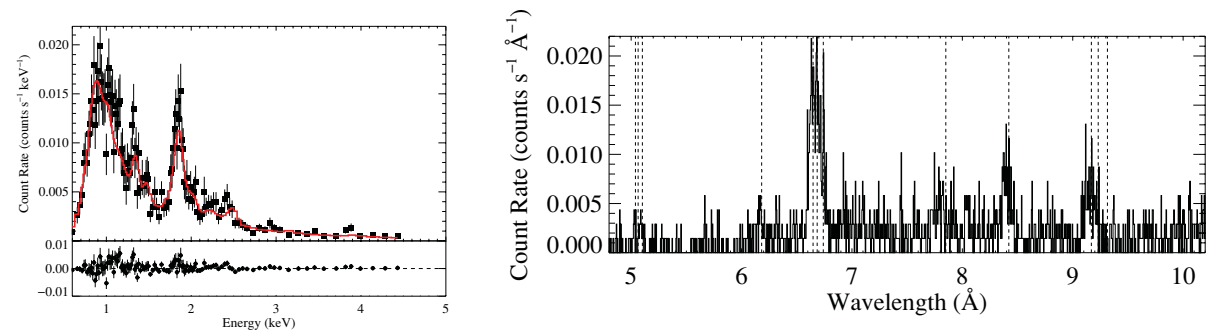

Figure 5. The zeroth-order, low-resolution Chandra spectrum of HD 93129A, with the best-fit thermal emission model attenuated by stellar wind absorption (left). This modeling shows that the emission temperature of the plasma is relatively low $-\mathrm{k} T=0.6 \mathrm{keV}$ - while the effects of wind attenuation are significant; explaining the observed hardness. The grating spectrum of this star is shown in the right-hand panel (dashed lines indicate rest wavelengths of important emission lines). Note that while the spectrum is quite hard (no significant emission longward of $10 \AA)$, the Si XIV Ly $\alpha$ line at $6.18 \AA$ is very weak compared to the Si xIII complex near $6.7 \AA$. This very low $\mathrm{H}$-like/He-like line ratio is indicative of plasma with a temperature of no more than 8 million $\mathrm{K}(0.7 \mathrm{keV})$.

account for the clumping). And furthermore, we find that when we model the broadband spectral properties and account for the effects of wind attenuation using a realistic radiation transport model in conjunction with a realistic opacity model, we derive similar mass-loss rate values.

Finally, we note that in this short paper we do not have the space to discuss in detail the possible role of porosity in generating the only modestly blue-shifted and asymmetric profiles. Porosity arises from clumping on very large scales, where individual clumps are optically thick to X-ray photoelectric absorption (Oskinova, Feldmeier, \& Hamann 2006). Our modeling suggests that porosity does not need to be invoked in order to explain the observed X-ray properties of the early O stars we discuss here. Their properties are well explained by modest mass-loss rate reductions. Furthermore, porosity requires clumping, by definition (but not the other way around). So, once clumping is invoked, and the density-squared diagnostics are adjusted accordingly, there is no longer any need to invoke porosity to explain the data. This and other aspects of porosity are addressed in the end-of-session discussion, later in these proceedings.

\section{References}

Cohen, D. H., Leutenegger, M. A., Wollman, E. E., Zsargó, J. et al. 2010, MNRAS, 405, 2391

Feldmeier, A., Puls, J. \& Pauldrach, A. W. A. 1997, A\&BA, 322, 878

Lamers, H. J. G. L. M. \& Leitherer, C. 1993, ApJ, 412, 771

Leutenegger, M. A., Cohen, D. H., Zsargó, J., Martell, E. M. et al. 2010, ApJ, 719, 1767

Markova, N., Puls, J., Repolust, T., \& Markov, H. 2004, A\&A, 413, 693

Oskinova, L. M., Feldmeier, A., \& Hamann, W.-R. 2006, MNRAS, 372, 313

Owocki, S. P., Castor, J. I., \& Rybicki, G. B. 1988, ApJ, 335, 914

Owocki, S. P. \& Cohen, D. H. 2001, ApJ, 559, 1108

Puls, J., Kudritzki, R.-P., Herrero, A., Pauldrach, A. W. A. et al. 1996, A\&SA, 305, 171

Puls, J., Markova, N., Scuderi, S., Stanghellini, C. et al. 2006, A\&A, 454, 625

Repolust, T., Puls, J., \& Herrero, A. 2004, A\&A A, 415, 349

Runacres, M. C. \& Owocki, S. P. 2002, A\&AA, 381, 1015

Smith, R. K., Brickhouse, N. S., Liedahl, D. A., \& Raymond, J. C. 2001, ApJ (Letters), 556, L91

Taresch, G., Kudritzki, R. P., Hurwitz, M., \& Bowyer, S. et al. 1997, A\&A A, 321, 531 